Journal of Case Reports 2018;8(4):279-281

\title{
Fibromatosis colli or Pseudotumour of Sternocleidomastoid
}

\author{
Saiyam Sahni ${ }^{1}$, Nikhil Mehrotra ${ }^{2}$, Rohit Singh ${ }^{3}$ \\ Departments of ${ }^{1}$ Radiology, ${ }^{2}$ Pediatrics, and ${ }^{3}$ Orthopaedics, Felix Hospital, Sector 137, Noida, Uttar Pradesh, India.
}

\section{Corresponding Author:}

Dr. Saiyam Sahni

Email: saiyam.sahni@gmail.com

This is an Open Access article distributed under the terms of the Creative Commons Attribution License (creativecommons.org/ licenses/by/3.0).

Received Accepted Published

May 12, 2018

October 25, 2018

December 10, 2018

\begin{abstract}
Background: Fibromatosis collis or pseudo-tumor of sternocleidomastoid in infancy is a rare cause of neck swelling in neonates. It is a congenital benign fibrous tumor developed from the sternocleidomastoid muscle, which if complicated, may lead to congenital torticollis. Case Report: Ultrasound of three week old neonate with right neck swelling showed fusiform thickening of the sternocleidomastoid muscle which showed heterogeneous echotexture. The maintained fibrillar structure, no vascular or bony evasion suggested fibromatosis collis. Conclusion: If diagnosed correctly, it can be managed conservatively with physiotherapy, and unnecessary investigations can be avoided.
\end{abstract}

Keywords: Congenital Torticollis, Fibroma, Infant, Neck Muscles, Physical Therapy Modalities.

\section{Introduction}

Fibromatosis colli manifests at birth or early neonatal period as neck swelling and sometimes torticollis. It has a prevalence of $0.4 \%$, with male predominance [1]. Though the exact etiology is not known, it is most likely due to birth trauma leading to ischemia of the muscle after difficult delivery. Though ultrasound is the imaging modality of choice, cross sectional imaging with computed tomography scan or magnetic resonance imaging may sometimes be required to further characterize the disease and to know the extent of involvement. [2]. We present a case report where fibromatosis colli was diagnosed using ultrasound in an infant.

\section{Case Report}

A three week-old neonate was referred to the radiology department for ultrasound of a right neck swelling that had been noticed by his mother four days ago. The child was otherwise healthy and there was no family history of lymphoma or tuberculosis. There was history of breech presentation and difficult delivery. On examination there was a preferential tilting of the head towards the left. A two $\mathrm{cm}$ firm non-mobile, non-tender swelling was seen in the anterior aspect of neck [Fig.1]. On ultrasound there was fusiform thickening of the sternocleidomastoid muscle which showed heterogeneous echotexture. The fibrillar structure of the muscle fibers was however maintained. No vascular invasion or bony involvement was seen [Fig.2,3]. There were few small benign appearing lymph nodes just distal to the muscle. The contralateral sternocleidomastoid muscle was normal in size, shape and echotexture. Both clinical and radiological findings favoured the diagnosis of fibromatosis colli.

A fine needle aspiration cytology of the lesion was done which showed spindle shaped cells with fibroblasts in a clean background. Besides these, we found a large number of muscle giant cells; the histopathology confirmed the diagnosis of fibromatosis colli. The parents were counselled and the infant was referred to physiotherapy for stretching exercises. The patient was reviewed after four weeks and there was significant reduction in the size of the neck swelling and normal neck movements were observed. 


\section{Discussion}

Fibromatosis colli is a benign fusiform mass of anterior neck arising from the sternocleidomastoid muscle. It usually presents few weeks after a traumatic delivery as a unilateral painless firm swelling mostly seen in the anterior neck [3]. Torticollis can develop in around $20 \%$ of cases. The investigation of choice is ultrasound which shows focal or diffuse thickening of the sternocleidomastoid muscle with homogenous or heterogenous echotexture. There is usually no associated cervical lymphadenopathy and no vascular invasion or bony involvement as may be seen with other inflammatory or malignant neck masses [4]. CT scan and MRI features have also been described. On CT scan, the sternocleidomastoid muscle appears diffusely enlarged, isodense in attenuation [5]. MRI features include decreased signal intensity of the mass on $\mathrm{T} 2 \mathrm{~W}$ images as compared to gradient-recalled $\mathrm{T} 1 \mathrm{~W}$ images, because of the presence of fibrous tissue [6].

\section{Conclusion}

Fibromatosis colli is a relatively rare cause of neck swelling in neonates and infants for which ultrasound is the diagnostic modality of choice and treatment is conservative.

Contributors: SS: manuscript writing, imaging and diagnosis; NM: manuscript editing, patient management; RS: critical inputs into the manuscript. SS will act as guarantor. All authors approved the final version of this manuscript. Funding: None; Competing interests: None stated.

\section{References}

1. Allouane MA, Elboussaadani A, Lezrag M, Zivkovic $\mathrm{V}$, Abada R, Roubal M, et al. Fibromatosis colli, a rare cause of neck mass in infants: a case report. J Case Rep Stud. 2016;4:407.

2. Smiti S, Kulkarni NM, Singh J. Case Report: Fibromatosis colli in a neonate. Indian Journal of Radiology. 2010;20(1):45-46.

3. Schneble F. Fibromatosis colli - sternocleidomastoid pseudotumor of infancy. Ped Rad [serial

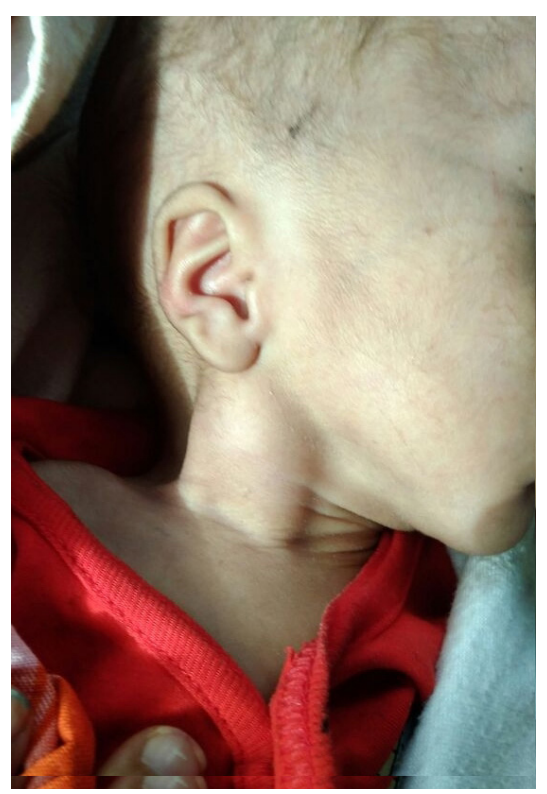

Fig.1: Infant with a firm swelling in the anterior aspect of neck with associated torticollis.

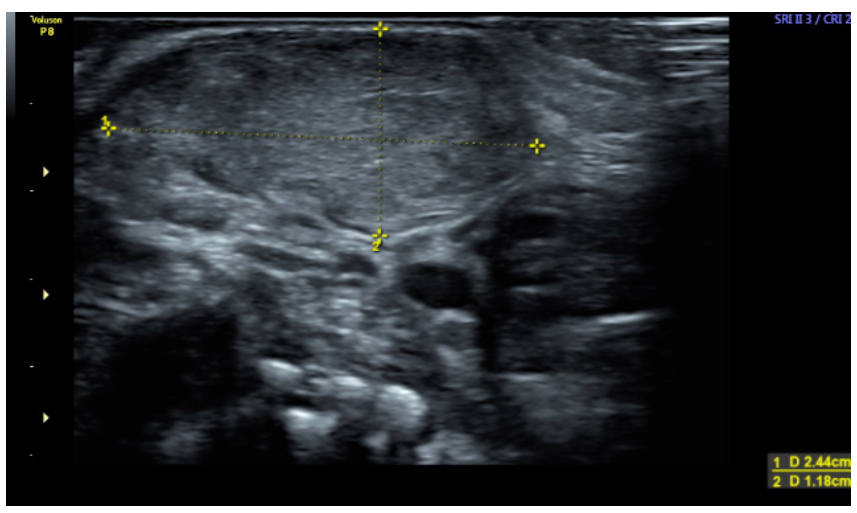

Fig.2: Fusiform enlargement of the right sternocleidomastoid muscle showing heterogeneous echotexture.

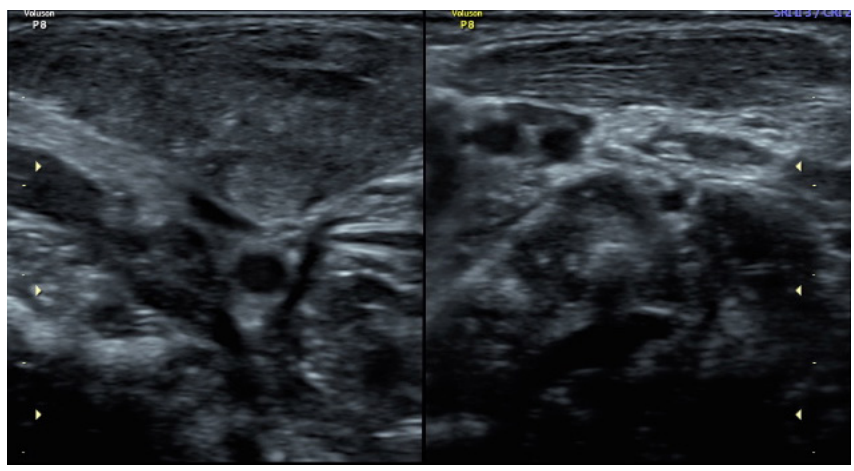

Fig.3: Longitudinal ultrasound image of the neck: Comparison of right sternocleidomastoid muscle with normal muscle on the left. 
online] vol 5 no. 6, Available at: www.PedRad. info/? search $=20050603161131$. Accessed on May $5^{\text {th }}$, 2018.

4. Sharma S, Mishra K, Khanna G. Fibromatosis colli in infants: A cytologic study of eight cases. Acta Cytol. 2003;47:359-362.

5. Crawford SC, Harnsberger HR, Johnson L, Aoki JR,
Alley J. Fibromatosis colli of infancy: CT and sonographic findings. AJR Am J Roentgenol. 1988;151:11811183.

6. Ablin DS, Jain K, Howell L, Steel D. West-ultrasound and MR imaging of fibromatosis Colli Pediatr Radiol. 1998;28:230-233. 